\title{
Effect of metal coating and residual stress on the resonant frequency of MEMS resonators
}

\author{
ASHOK KUMAR PANDEY, K P VENKATESH and RUDRA \\ PRATAP* \\ CranesSci MEMS Laboratory, Department of Mechanical Engineering, Indian \\ Institute of Science, Bangalore 560012 \\ e-mail: pratap@mecheng.iisc.ernet.in
}

\begin{abstract}
MEMS resonators are designed for a fixed resonant frequency. Therefore, any shift in the resonant frequency of the final fabricated structure can be a denting factor for its suitability towards a desired application. There are numerous factors which alter the designed resonant frequency of the fabricated resonator such as the metal layer deposited on top of the beam and the residual stresses present in the fabricated structure. While the metal coating, which acts as electrode, increases the stiffness and the effective mass of the composite structure, the residual stress increases or decreases the net stiffness if it is a tensile or compressive type respectively. In this paper, we investigate both these cases by taking two different structures, namely, the micro cantilever beam with gold layer deposited on its top surface and the MEMS gyroscope with residual stresses. First, we carry out experiments to characterize both these structures to find their resonant frequencies. Later, we analytically model those effects and compare them with the experimentally obtained values. Finally, it is found that the analytical models give an error of less than $10 \%$ with respect to the experimental results in both the cases.
\end{abstract}

Keywords. Resonator; metal coating; residual stress; experimental results; analytical model.

\section{Introduction}

With the increasing use of microengineering techniques (Madou 1997) in producing sensing and actuating devices, it become increasingly significant to consider various after-effects of these techniques if the devices are expected to perform to their specification. MEMS resonators made of polysilicon have potential application in the areas of RF-MEMS (Senturia 2000), MEMS oscillators (Maluf 2000), resonant sensors such as biological mass detector (Craighead 2000), etc. As a resonator, the most important characteristics are the resonant frequency and the quality factor (Fedder 1994, Rao 1995). Since the MEMS resonators are designed for a fixed resonant frequency, any shift in the resonant frequency of the final fabricated structure can be a denting factor for its suitability towards a desired application. There are numerous

*For correspondence 
factors which alter the designed resonant frequency of the fabricated resonator. Some of these factors are the metal layer which is deposited on the top of the structural layer and the presence of residual stresses in the fabricated structure. The metal layer coating, which acts as an electrode, increases the stiffness and the effective mass of the composite structure (Chiou \& Lin 2005, Zhang et al 2005) while the residual stress increases or decreases the net stiffness if it is a tensile or compressive stresses respectively (Zhangt et al 1990, Bellout et al 1999, Pandey et al 2007).

There are many studies which deal with modelling the effect of metal coating and residual stresses on the resonant frequencies of simple devices. Chiou \& Lin (2005) have proposed the computation of effective flexural rigidity in the design of a composite cantilever beam. Zhang et al (1990) have also found the analytical solution for predicting effective stiffness and residual stresses in multilayered coatings in MEMS structure. Zhangt et al (1990) have proposed the resonant frequency technique to find the elastic constants of the silicon beam from the experimental results. The resonant frequency technique has been described in (Bellout et al 1999) for fixed-fixed beam to compute elastic constants and residual stresses. The frequency resonant technique for the guided cantilever beam is also presented in (Pandey et al 2007).

In this paper, we investigate the effects of metal coating as well as residual stress on the resonance frequencies by taking two different structures. The effect of metal coating is studied by taking a cantilever beam which is fabricated using PolyMUMPs process (MUMPs) as

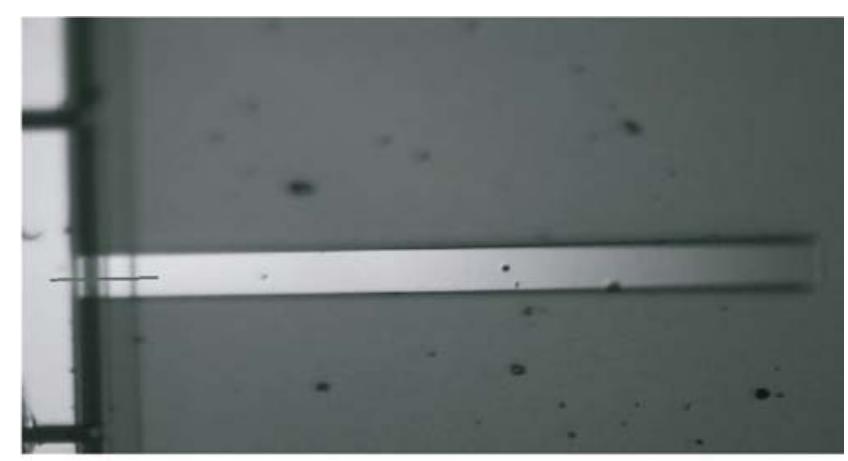

(a)

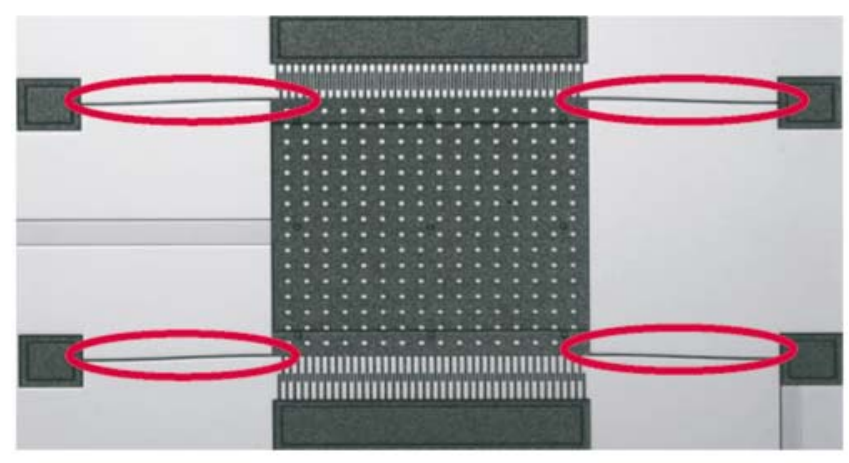

(b)
Figure 1. (a) Micro cantilever beam and (b) MEMS gyroscope. 
Table 1. Geometric dimensions of the structure.

\begin{tabular}{lll}
\hline Structure & Parameter & Value \\
\hline \multirow{3}{*}{ Cantilever } & Beam length, $l_{b}$ & $390 \mu \mathrm{m}$ \\
& Beam width, $W_{b}$ & $30 \mu \mathrm{m}$ \\
& Poly silicon thickness & $2 \mu \mathrm{m}$ (ideal) \\
& Metal thickness & $0.5 \mu \mathrm{m}$ (ideal) \\
& Proof mass & $500 \times 500 \mu \mathrm{m}^{2}$ \\
Gyro & Beam length, $l_{b}$ & $300 \mu \mathrm{m}$ \\
& Beam width, $W_{b}$ & $4 \mu \mathrm{m}$ \\
& Hole size & $10 \times 10 \mu \mathrm{m}^{2}$ \\
& Thickness & $3.5 \mu \mathrm{m}$ \\
\hline
\end{tabular}

shown in figure 1a. The effect of residual stress is studied by taking another MEMS resonator (fabricated using surface micromachining process under different operating conditions) which consists of a proof mass and is supported by four guided cantilever beams as shown in figure $1 \mathrm{~b}$. We then characterize both these structures using Polytec Scanning Laser Vibrometer (PSV) a Polytec product (Polytec) for the out-of-plane motion - to find their resonant frequencies. Finally, we analytically model the effects of metal layer and the residual stress on the resonant frequency for both the structures and then compare the results with the experimental values.

\section{Microfabrication process}

The PolyMUMPs process (Madou 1997) which is used to fabricate cantilever resonator has different variations in the thickness of the metal and the structural layers, and also the Young's modulus of the polysilicon layer. In this process, the thickness may vary from $1.85 \mu \mathrm{m}$ to $2.15 \mu \mathrm{m}$ for the polysilicon layer and $0.46 \mu \mathrm{m}$ to $0.58 \mu \mathrm{m}$ for the metal layer (MUMPs). The structure micro cantilever beam shown in figure $1 \mathrm{a}$ is free from residual stresses. Its important dimensions are mentioned in table 1.

Another MEMS structure, shown in figure $1 \mathrm{~b}$, is fabricated under different operating conditions. The fabrication of this device also involves the well-known steps of microfabrication (Madou 1997). This structure seems to be affected by the presence of residual stress. This fact can be assessed by observing the SEM image of the fabricated structures on the same silicon wafer in which some of the structures are permanently deflected as shown in figure $1 \mathrm{~b}$. The important dimensions of the fabricated structure are mentioned in table 1.

\section{Experimental studies}

To excite and measure the dynamic characteristics of the structures, we use MSA 400 Microsystem Analyzer - a Polytec product (Polytec) to characterize the out-of-plane vibrations by Scanning Laser-Doppler Vibrometer which works on the principle described in figure 2. The working principle involves electrical excitation which causes the suspended structure to vibrate with respect to the substrate. The measurement laser beam from the interferometer in the scanning head is positioned to a scan point on the object by means of mirrors 


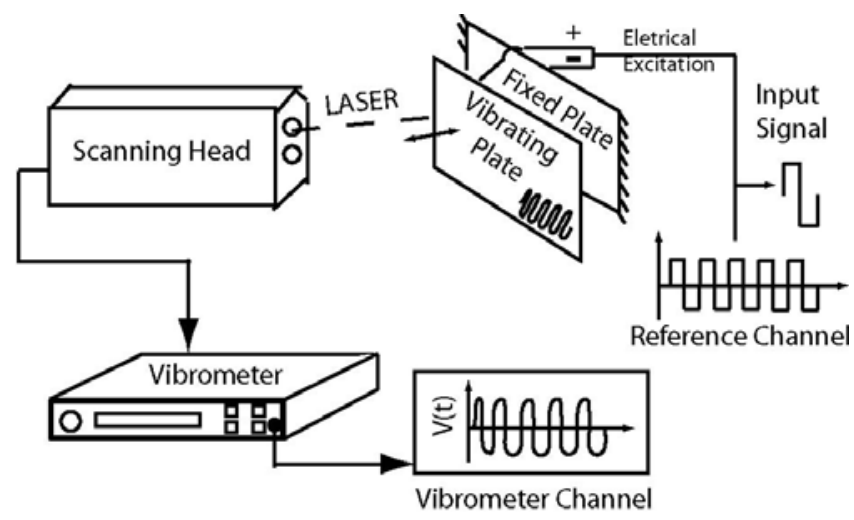

Figure 2. Working principle of test set-up.

and is scattered back. The back-scattered laser light interferes with the reference beam in the scanning head. A photo detector records the interference. A decoder in the vibrometer provides a voltage which is proportional to the velocity of the vibration parallel to the measurement beam. The voltage is digitized and processed as vibrometer signal. Based on the above principle, the measurement details of the micro cantilever beam and micromachined gyroscope are described in the following two sections.

\subsection{Measurements for cantilever beam}

To find the resonant frequencies of the microcantilever structures, we apply $1 \mathrm{~V} \mathrm{DC}$ and $0.6 \mathrm{~V}$ $\mathrm{AC}$ pseudorandom signal with frequencies ranging from $1 \mathrm{kHz}$ to $1 \mathrm{MHz}$. From the captured modes and the frequencies shown in figure 3 , the resonant frequencies of cantilever beam of length $390 \mu \mathrm{m}$ are found and mentioned in table 2 . The response curves and the mode shapes corresponding to first five bending modes are also shown in figure 3.

\subsection{Measurements for gyroscope}

To find the resonant frequency corresponding to the out-of-plane mode of the MEMS gyroscope (shown in figure 1b), we apply $4 \mathrm{~V} \mathrm{DC}$ and $1 \mathrm{~V} \mathrm{AC}$ pseudorandom signal with frequen-

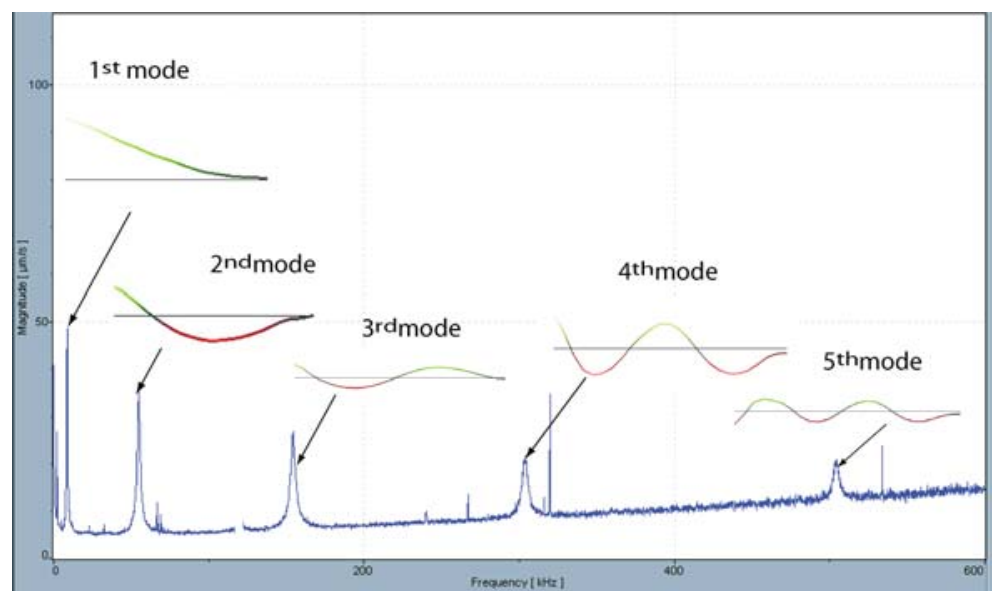

Figure 3. Response of cantilever with gold coating. 
Table 2. Experimental results for cantilever beam.

\begin{tabular}{cc}
\hline Mode number & Frequency in $\mathrm{kHz}$ \\
\hline 1 & $8 \cdot 75$ \\
2 & $54 \cdot 84$ \\
3 & $154 \cdot 2$ \\
4 & $304 \cdot 1$ \\
5 & $504 \cdot 5$ \\
\hline
\end{tabular}

cies ranging from $1 \mathrm{kHz}$ to $40 \mathrm{kHz}$. The captured mode and the frequency corresponding to the out-of-plane motion are shown in figure 4 . The fundamental resonant frequency is found to be $16.9 \mathrm{kHz}$.

\section{Analytical models and their comparison with experimental results}

In the first part of the section, we analytically compute the resonant frequency of the cantilever beam which is made of $2 \mu \mathrm{m}$ thickness polysilicon material and is coated with $500 \mathrm{~nm}$ thickness of gold layer. In the second part of the paper, the effect of residual stress is analytically modelled for the MEMS gyroscope. Finally, the analytical results are compared with the experimental results in the corresponding sections.

\subsection{Effect of metal coating}

Figure 3 shows the response of the microcantilever beam which is coated with $500 \mathrm{~nm}$ gold layer. In this section, we provide an analytical formulation to model the effect of metal coating on the resonant frequency of a cantilever beam.

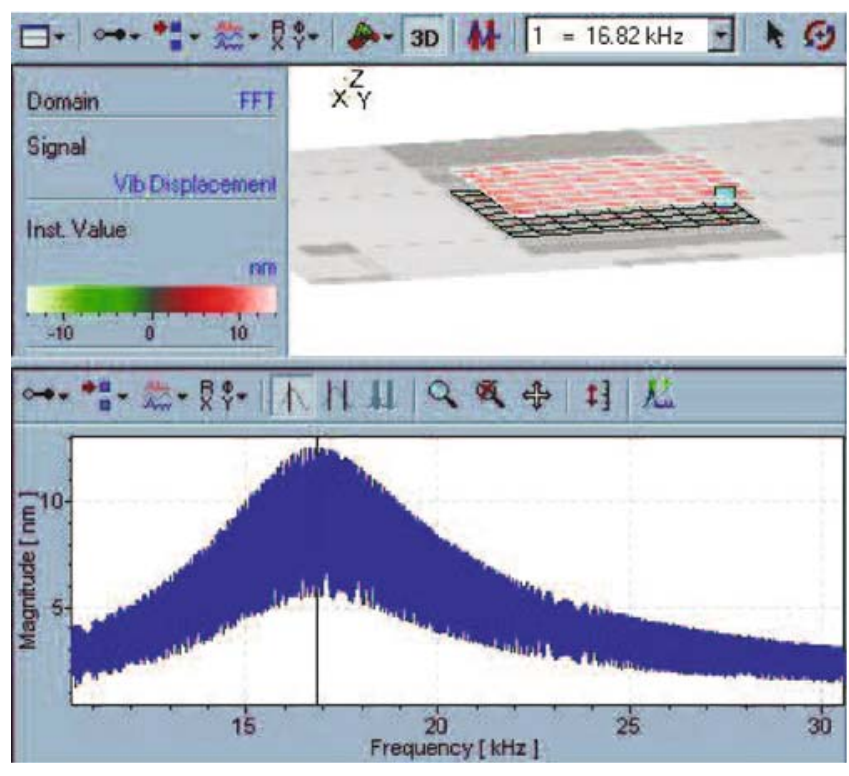

Figure 4. Frequency response of MEMS gyro. 


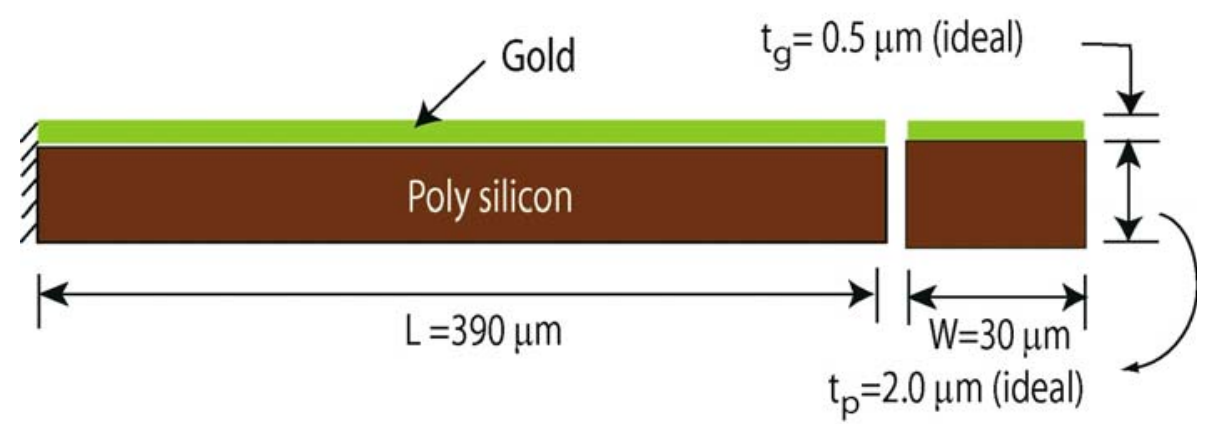

Figure 5. Polysilicon beam with gold coating.

Figure 5 shows the pictorial representation of polysilicon cantilever beam with a gold coating of $0.5 \mu \mathrm{m}$ if the fabrication condition is taken as an ideal condition. Since there ought to be the variation in the thickness of the final structure which is fabricated from PolyMUMPs process, the following variations in the thickness of polysilicon and the gold layer are taken into account: $1.85 \mu \mathrm{m}$ to $2.15 \mu \mathrm{m}$ with an ideal value of $2.0 \mu \mathrm{m}$ for polysilicon layer and $0.46 \mu \mathrm{m}$ to $0.58 \mu \mathrm{m}$ with an ideal value of $0.52 \mu \mathrm{m}$ for the gold layer (mumps). Because of different operating conditions of the PolyMUMPs process, the Young's modulus of the polysilicon film is also found to be in the range of $158 \pm 10 \mathrm{GPa}$ (mumps). Now, to model the effect of metal coating on resonant frequencies, we start with the computation of effective flexural rigidity of the beam in the subsequent section.

4.1a Effective flexural rigidity calculations: Since the displacement about the neutral axis remains the same in both the polysilicon and gold films, it can be treated as two springs in parallel. Let us assume $E_{P}$ as the Young's modulus of polysilicon and $E_{g}$ as that of gold. Similarly, $I_{P}$ as the moment of inertial of polysilicon film about the neutral axis and $I_{g}$ as that of gold film. Then, the effective flexural rigidity, $E I$, may be computed from

$$
(E I)_{\mathrm{eff}}=E_{p} I_{p}+E_{g} I_{g} .
$$

Therefore, the computation of flexural rigidity of the composite beam involves the determination of moment of inertia of the polysilicon structure and the gold layer about its neutral axis. Referring to figure 6 , the effective moment of inertia of the polysilicon beam about the neutral axis of the composite structure is given by:

$$
I_{p}=\frac{W t_{p}^{3}}{12}+W t_{p}\left(\frac{H}{2}-\frac{t_{p}}{2}\right)^{2} .
$$

And that of gold layer is given by

$$
I_{g}=\frac{W t_{g}^{3}}{12}+W t_{g}\left(\frac{H}{2}-\frac{t_{g}}{2}\right)^{2} .
$$

Substituting the values of moment of inertia of polysilicon film and gold film in eqn. (1), we get an effective flexural rigidity for the Young's modulus of polysilicon as $E_{p}=160 \mathrm{GPa}$ and that of gold as $E_{g}=77 \mathrm{GPa}$. The value of effective Young's modulus of the composite structure is found to be $85.5 \mathrm{GPa}$ for a given $I=\frac{W t_{g}^{3}}{12}$. 


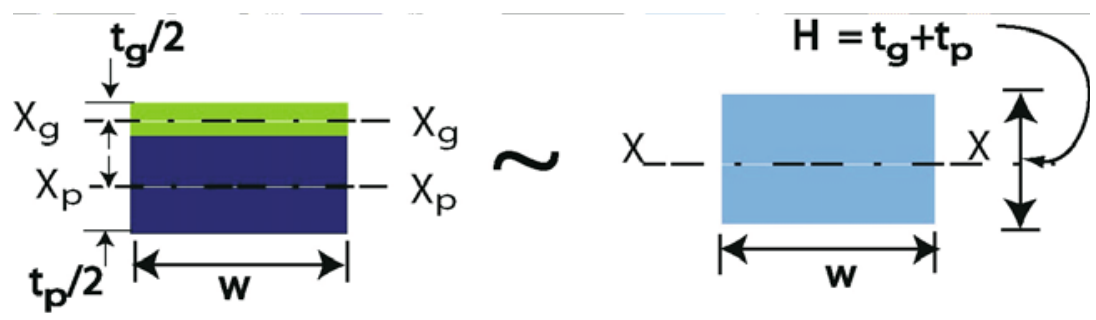

Figure 6. Equivalent beam model for composite beam.

4.1b Computation of resonant frequency: The resonant frequency of cantilever beam is given by (Rao 1995):

$$
\omega=C \sqrt{\frac{E I}{\rho l^{4}}} .
$$

Where $\rho$ is the linear density (mass per unit length) of a composite beam made of polysilicon and gold layers. It is given by:

$$
\rho=\frac{M_{\text {poly Si }}+M_{\text {gold }}}{l},
$$

$l$ is the length of the beam and $C$ is a constant which depends on different modes as mentioned in table 3 (Rao 1995). The values of $E I$ are computed from eqn. (1).

The third column of table 3 presents the first five resonant frequencies $f_{w m}$ of the polysilicon cantilever beam without any metal coating. The fourth column presents the resonant frequency $f_{m}$ of the composite beam in which the gold layer is deposited on the top of the polysilicon beam. These frequencies are computed from eqn. (4). If we compare the ratio of the frequencies $f_{w m}$ and $f_{m}$ for different modes, it is found that $f_{w m} / f_{m} \approx 2 \cdot 2$. This difference is mainly due to a reduction in the effective flexural rigidity and an increase in the effective mass of the system because of gold layer on the top of polysilicon beam resonator.

4.1c Comparison between analytical and experimental results: Table 4 compares the analytical and experimental results of the cantilever beam with thickness of $1.85 \mu \mathrm{m}$ for

Table 3. Values of $C$ and the resonant frequencies for different modes of the cantilever beam with and without metal coating.

\begin{tabular}{cccr}
\hline Mode number & \multicolumn{1}{c}{$C$} & $f_{\mathrm{wm}}$ (in kHz) & $f_{m}$ (in kHz) \\
\hline 1 & 3.516 & 19.7 & 9.35 \\
2 & 22.036 & 123.4 & 58.63 \\
3 & 61.697 & 345.4 & 164.17 \\
4 & 120.902 & 676.8 & 321.71 \\
5 & 199.86 & 1118.9 & 531.82 \\
\hline
\end{tabular}


Table 4. Comparison between analytical and experimental results.

\begin{tabular}{ccc}
\hline Mode number & $f_{\text {anal }}$ & \multicolumn{1}{c}{$f_{\exp }$} \\
\hline 1 & $9.35 \mathrm{kHz}$ & $8.75 \mathrm{kHz}$ \\
2 & $58.63 \mathrm{kHz}$ & $54.84 \mathrm{kHz}$ \\
3 & $164.17 \mathrm{kHz}$ & $154.2 \mathrm{kHz}$ \\
4 & $321.71 \mathrm{kHz}$ & $304.1 \mathrm{kHz}$ \\
5 & $531.82 \mathrm{kHz}$ & $504.5 \mathrm{kHz}$ \\
\hline
\end{tabular}

polysilicon material and $0.46 \mu \mathrm{m}$ for gold layer. It is found that the analytical results give an error of less than $5 \%$ for first five modes.

If we take an admissible variation in the thicknesses of the polysilicon and gold layers, we get different values of resonant frequencies. Table 5 provides an error between the analytical and experimental results for different thicknesses of composite cantilever beam. It is found that the percentage error of the analytical results may vary from $5 \%$ to $22 \%$ depending on the admission thickness of the cantilever beam fabricated using PolyMUMPs process.

\subsection{Effect of residual stress}

To study the effect of residual stress, we take MEMS gyroscope. Figure 4 shows the response of MEMS gyroscope structure in its out-of-plane motion. The analytical modelling of this effect in this case may be done by considering the equivalent spring-mass model of the original system. In this structure, the cantilever beams which are used to support the proof mass are assumed to be a guided beam.

In this paper, an approximate value of the fundamental resonant frequency is obtained from the energy method (Rao 1995, Zhangt et al 1990). This method equates the kinetic energy due to oscillation with the potential energy due to both deflection and axial stress and then solves for the natural frequency. Rayleigh's quotient (i.e. the ratio of potential energy to kinetic energy) for MEMS gyroscope structure which consists of a proof mass and four axially loaded guided beams is given by (Bellout et al 1999, Pandey et al 2007):

$$
\omega^{2}=\frac{4 E I \int_{0}^{l} \frac{d^{2} y}{d x^{2}} d x+4 \sigma A \int_{0}^{l} \frac{d^{2} y}{d x^{2}} d x}{4 \rho A \int_{0}^{l} y^{2} d x+m_{\text {plate }}},
$$

Table 5. Error between analytical and experimental results for different values of polysilicon and gold layers thickness.

\begin{tabular}{ccc}
\hline$t_{p}$ & $t_{g}$ & {$\left[\left(f_{a}-f_{\text {exp }}\right) / f_{a}\right] \times 100$} \\
\hline $1.85 \mu \mathrm{m}$ & $0.46 \mu \mathrm{m}$ & $5 \%$ \\
$2.00 \mu \mathrm{m}$ & $0.52 \mu \mathrm{m}$ & $15 \%$ \\
$2.15 \mu \mathrm{m}$ & $0.58 \mu \mathrm{m}$ & $21 \%$ \\
\hline
\end{tabular}


where $E$ is the Young's modulus of the polysilicon material, $\rho$ is the density of polysilicon, $I$ is the area moment of inertia, $A$ is the cross-sectional area, $\sigma$ is the axial residual stress in the released structure, $m_{\text {plate }}$ is the mass of the perforated plate, and $y(x)$ is the mode shape of oscillating beam. Let us assume the mode shape of the guided cantilever beam as (Pandey et al 2007):

$$
y(x)=\frac{1}{2}-\frac{1}{2} \sin \left(\frac{\pi x}{l}\right) .
$$

Substituting the mode shape from eqn. (7) in eqn. (6), the simplified form of the first natural frequency of the MEMS gyroscope is given by:

$$
\omega^{2}=\frac{\pi^{4}}{2} \frac{E I}{l^{3} m_{\mathrm{eff}}}+\frac{\pi^{2}}{2} \frac{\sigma A}{l m_{\mathrm{eff}}},
$$

where $m_{\text {eff }}=\frac{13}{35} \rho A l+m_{\text {plate }}$ is the effective mass of the structure, $m_{\text {plate }}=1.87 \mu g$ is the mass of the perforated proof mass. The first term of eqn. (8) is the first natural frequency of the MEMS gyroscope without residual stress.

To compute the residual stress and the resultant Young's modulus in the MEMS structure shown in figure $1 \mathrm{~b}$, we consider two different structures $A$ and $B$. For these structures, $l_{A}=300 \mu \mathrm{m}$ and $l_{B}=400 \mu \mathrm{m}$ are the length of the guided cantilever beams. The cross-sectional properties, material properties, and residual stresses are same in both the structures. If $f_{A}$ and $f_{B}$ are resonant frequencies of the structure $A$ and $B$, respectively, then the expression for Young's modulus and residual stress can be obtained from eqn. (8):

$$
\begin{aligned}
& E=\frac{8}{\pi^{2} I} \frac{l_{A} m_{\mathrm{eff}}^{A} f_{A}^{2}-l_{B} m_{\mathrm{eff}}^{B} f_{B}^{2}}{\left(\frac{1}{l_{A}^{2}}-\frac{1}{l_{B}^{2}}\right)} \\
& \sigma=8 \frac{l_{A}^{3} m_{\mathrm{eff}}^{A} f_{A}^{2}-l_{B}^{3} m_{\mathrm{eff}}^{B} f_{B}^{2}}{l_{A}^{2}-l_{B}^{2}} .
\end{aligned}
$$

Now, for the structure $A$ and $B$ of beam lengths $l_{A}=300 \mu \mathrm{m}$ and $l_{B}=400 \mu \mathrm{m}$, respectively, the experimental values of the first resonant frequency is obtained as $f_{A}=16.9 \mathrm{kHz}$ and $f_{B}=14.1 \mathrm{kHz}$. Taking the cross-sectional area $A_{c s}=14.0 \mathrm{mum^{2 }}$, the area moment of inertia $I=1.42 \times 10^{-23} \mathrm{~m}^{4}$ and the density $\rho=2300 \mathrm{~kg} / \mathrm{m}^{3}$, the values of $E$ and $\sigma$ are given by:

$$
E=131 \cdot 11 \mathrm{GPa} \quad \text { and } \quad \sigma=77.71 \mathrm{MPa} .
$$

If we neglect the effect of residual stress in the formula, we end up getting different values of the natural frequency, i.e. $f_{300}^{0}=6.74 \mathrm{kHz}$ and $f_{400}^{0}=4.37 \mathrm{kHz}$ which are an order of magnitude less than the actual values of $16.9 \mathrm{kHz}$ and $14.1 \mathrm{kHz}$, respectively. Therefore, it is essential to include the effect of residual stress developed due to fabrication process in order to predict the actual frequency of the released structure. The difference in the resonant frequency of the stressed and unstressed structures may also be obtained from the numerical simulation in ANSYS as shown in figure 7. 


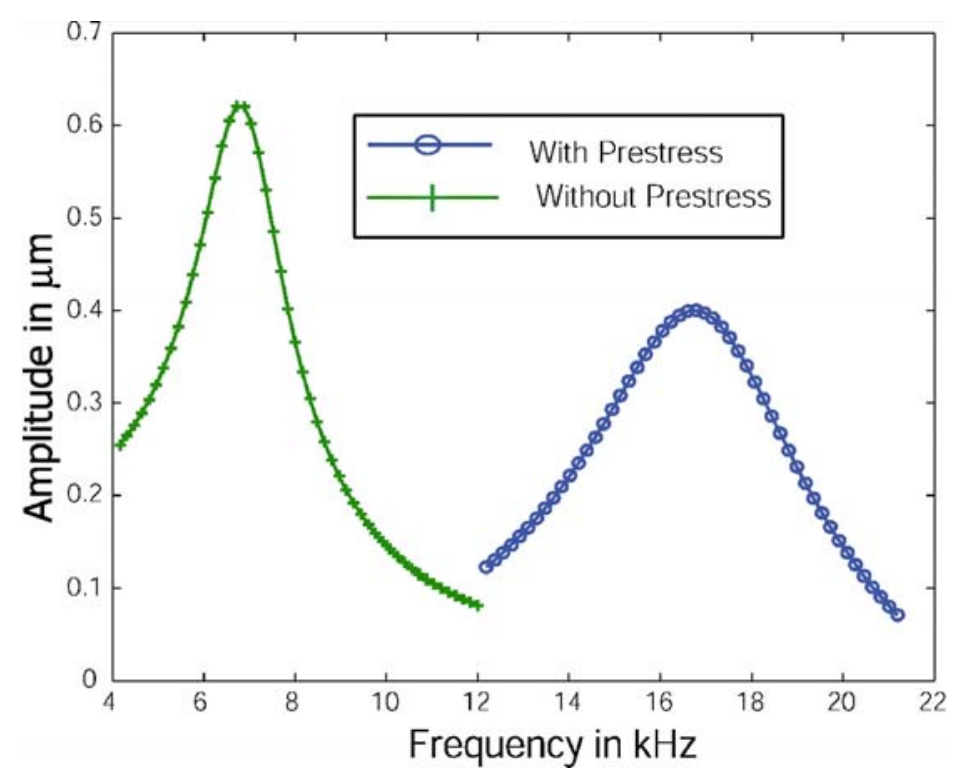

Figure 7. Shift in resonant frequency due to tensile residual stress.

\section{Conclusions}

The effect of metal coating on the resonant frequency is studied by taking a MEMS cantilever while the effect of residual stress is studied with MEMS gyroscope. The experiments are performed to find out their resonant frequencies. The analytical models are also presented to model these effects.

For the MEMS cantilever beam which is found to be in unstressed state, the resonant frequency changes by $15 \%$ if the thickness of the polysilicon and the gold layers are allowed to change from $1.85 \mu \mathrm{m}$ to $2.15 \mu \mathrm{m}$ and $0.46 \mu \mathrm{m}$ to $0.58 \mu \mathrm{m}$, respectively. Since the Young's modulus of the poly layer is also a variant of the process parameters, the frequency change of 6 to $8 \%$ is observed for the Young's modulus of $158 \pm 10 \mathrm{GPa}$. For the polysilicon and metal layers of thicknesses $1.85 \mu \mathrm{m}$ and $0.46 \mu \mathrm{m}$, respectively, and the Young's modulus of the polysilicon as $160 \mathrm{GPa}$, the analytical result gives an error of $5 \%$ with respect to the experimental result. In the study of the effect of residual effect, it is found that tensile residual stress of $77.71 \mathrm{MPa}$ changes the natural frequency from $6.77 \mathrm{kHz}$ to $16.97 \mathrm{kHz}$ in MEMS gyroscope. These changes are also verified using numerical simulation in ANSYS.

This work was funded by the Department of Science and Technology, New Delhi, India and National Program of Smart Materials, Bangalore. We acknowledge the contribution of Prof. P S Gandhi of Indian Institute of Technology Bombay, India, for the fabricated microcantilever beam. 


\section{References}

Bellout H, Neustupa J, Penel P 1999 Elastic properties and vibration of micro-machined structures subject to residual stresses. Proc. IEEE Canadian Conference on Electrical and Computer Engineering, 1674-1679

Chiou J C and Lin Y J 2005 A novel large displacement electrostatic actuator: Prestress comb drive actuator. J. Micromech. Microeng. 15: 1641-1648

Craighead H G 2000 Nanoelectromechanical systems. Science 290(5496): pp. 1532-5

Fedder G K 1994 Simulation of microelectomechanical systems. Ph.D thesis, University of California.

Madou M 1997 Fundamentals of microfabrication, (Florida: CRC Press).

Maluf N 2000 Introduction to microelectromechanical systems engineering, (Boston: Artech house)

Pandey A K, Rudra Pratap, Venkatesh K P 2007 Effect of residual stress and perforations on the dynamic characterstics of MEMS devices. Proc. $1^{\text {st }}$ National Conference of Research Scholars in Mechanical Engineering, India, 35-40

Polytec reference manual. URL: http://www.polytec.com

Rao S S 1995 Mechanical Vibrations, (New York: Wesley Publishing Company)

Senturia S D 2000 Microsystem Design, (Boston: Kluwer Academic Publishers)

URL: http://www.memsrus.com/documents/polyMUMPS. rundata.xls

Zhang X C, Xu B S, Wang H D, Wu Y X 2005 An analytical model for predicting thermal residual stresses in multilayer coating. J. Thin Solid Films 488: 274-282

Zhangt L M, Zhangt, Uttamchandanit D, Culshawt B, Dobson P 1990 Measurement of Young's modulus and internal stress in silicon microresonators using a resonant frequency technique. Meas. Sci. Technol. 1: 1343-1346 